\title{
Late-Developing Metastatic Malignant Melanoma in the Thoracic Spine Originating from Choroidal Melanoma
}

\author{
Jun Kyu Hwang ${ }^{1}$, \\ Kyung Hyun $\mathrm{Kim}^{1}$, \\ Ji Sup Kim², \\ Sung Jun $\mathrm{Ahn}^{3}$, \\ Sung Uk Kuh ${ }^{1}$ \\ Departments of ${ }^{1}$ Neurosurgery, \\ ${ }^{2}$ Pathology, and ${ }^{3}$ Radiology, \\ Gangnam Severance Hospital, \\ Spine and Spinal Cord Institute, \\ Yonsei University College of \\ Medicine, Seoul, Korea
}

\section{Corresponding Author:}

\section{Sung Uk Kuh}

Department of Neurosurgery, Gangnam Severance Hospital, Spine and Spinal Cord Institute, Yonsei University College of Medicine, 211 Eonju-ro, Gangnam-gu, Seoul 06273, Korea

Tel: +82-2-2019-3390

Fax: +82-2-3461-9229

E-mail: kuhsu@yuhs.ac

Received: March 12, 2017

Revised: June 5, 2017

Accepted: June 7, 2017
Copyright (C) 2017 by The Korean Spinal Neurosurgery Society

This is an open access article distributed under the terms of the Creative Commons Attribution Non-Commercial License (http://creativecommons.org/licenses/bync/4.0/) which permits unrestricted noncommercial use, distribution, and reproduction in any medium, provided the original work is properly cited.

A 54-year-old woman visited Gangnam Severance Hospital for left side flank pain. She had a history of total removal of malignant melanoma on the left eye ball 20 years prior. No evaluation had been performed since then. A paravertebral mass at thoracic ninth level (T9) was discovered on spinal magnetic resonance imaging, and pathology confirmed malignant melanoma. Following positron emission tomography-computed tomography, no other metastasis was discovered. After removal of the paravertebral mass, palliative chemotherapy (dacabarzine + tamoxifene) was administered in 3 cycles over 2 months. Radiotherapy with simultaneous integrated boost technique was performed at 4,350 cGy total over 15 days, 290 cGy per delivery, and was administered with the first cycle of palliative chemotherapy. Despite this treatment, multiple metastases developed throughout her body 7 months later, and the patient is continuing chemotherapy.

Key Words: Choroidal tumor, Malignant melanoma, Spine metastasis

\section{INTRODUCTION}

Malignant melanoma is tumor of poor prognosis originating from melanocytes located in the skin or mucosa. Melanoma metastasizes to many sites including liver, bone, lung, and lymph nodes. With metastasis, the 5-year survival rate is $5 \%-14 \%$. Malignant melanoma from skin is the most fatal form ${ }^{7}$. Historically, prognosis of such patients has been poor, with death occurring 6-12 months from the first metastasis $^{10)}$. Despite many advances in the diagnosis and treatment of primary tumors in recent years, there has been no improvement in survival rates, and the median survival time following diagnosis with metastasis remains poor ${ }^{4}$.

Uveal melanoma is the most frequent malignant tumor of the eye ball, the primary treatments for which are surgical resection, chemotherapy, and radiotherapy. This case report describes a patient with long-term survival over a period of 20 years from first diagnosis without recurrence or metastasis. Although no other systemic treatment was administered, the patient lived without metastasis or specific symptoms. However, 20 years after diagnosis, the first metastasis was detected as a paravertebral focus (thoracic ninth level), and multiple metastases occurred within the next several months.

\section{CASE REPORT}

A 54-year-old woman visited Gangnam Seve- rance Hospital with left flank pain of 2 months duration. There was a history of enucleation of the left eye ball 20 years prior, and pathology demonstrated malignant melanoma. Per the past surgical report, there was a $1.5-\times 1-\mathrm{cm}$-sized darkish mass at the subretinal area of the left eye ball, extending focally to the sclera, although the optic nerve resection margin was clear.

On the current workup, preoperative T-spine enhanced computed tomography demonstrated a dumbbell-shaped enhanced mass (Fig. 1). On T-spine magnetic resonance imaging, a high intensity signal was noted at the paravertebral thoracic ninth level in the T1-weighted image, but a low intensity signal was detected in this area on T2-weighted image (Fig. 2). Positron emission tomography-computed tomography revealed no other metastasized lesion (Fig. 3). Tumor removal at the thoracic level was performed with pedicle screw fixation of T7-8-9-1011 right and T7-8-11 left. Twenty-five days after total removal of the T9 lesion (Figs. 4-6), the first cycle of palliative chemotherapy (dacabarzine + tamoxifene) was initiated and involved three complete cycles over 2 months. Radiotherapy with simultaneous integrated boost technique was performed for a total of 4,350 cGy over 15 days (290 cGy per delivery episode) and was administered with the first cycle of palliative chemotherapy. Multiple metastases were discovered on whole body bone scan seven months after paravertebral metastasis resection (Fig. 7). 

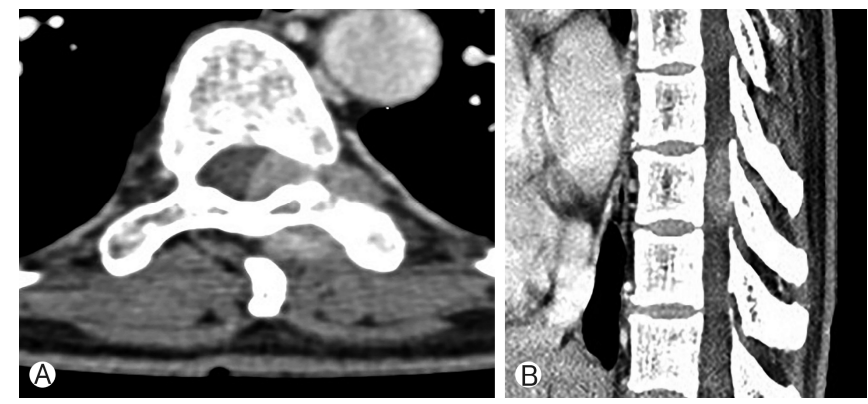

Fig. 1. Preoperative computed tomography (CT). On axial view (A) and sagittal view (B) of T-spine contrast-enhanced CT, a strongly enhanced mass is observed involving the T9 spinal canal, posterior column of vertebra, and adjacent soft tissue. Associated bony destruction is seen at the left transverse process.
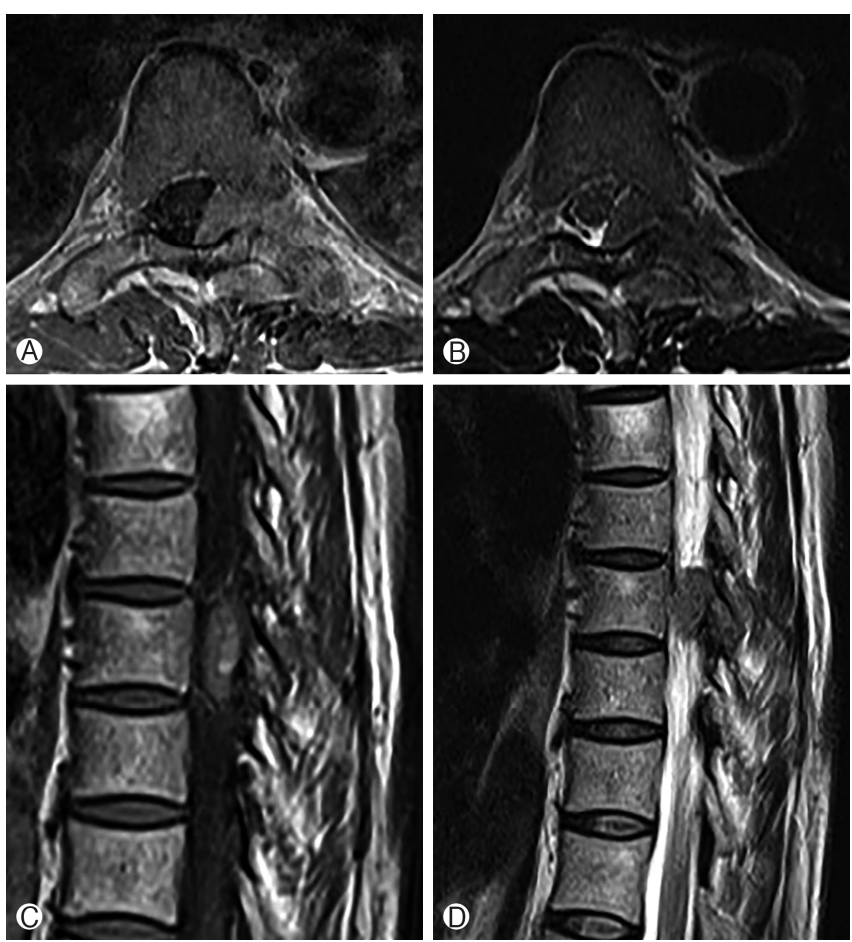

Fig. 2. Preoperative magnetic resonance imaging (MRI). On axial (A) and sagittal images (C) of gadolinium-enhanced MRI, strongly enhanced tumor is observed, involving the T9 spinal canal, posterior column of vertebra, and adjacent soft tissue. On axial (B) and sagittal views (D) of T2-weighted image, the lesion shows iso signal intensity.

\section{DISCUSSION}

Malignant melanoma is a cancer of poor prognosis because of the high rates of multiple metastases. There are rare cases of long-term survival longer than 20 years from first diagnosis, as seen in our case. There was a similar case at Kobe University.

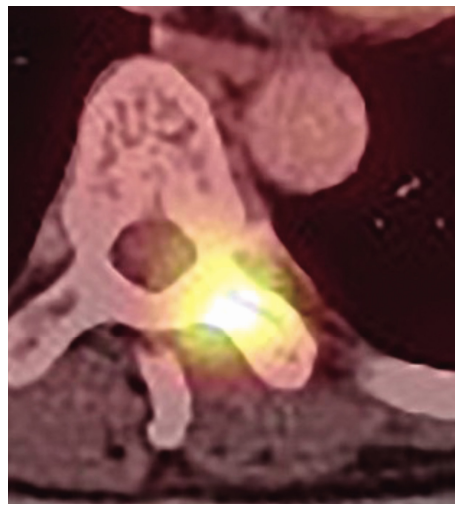

Fig. 3. Preoperative positron emission tomography-computed tomography whole body (Torso) Focal intense fluorodeoxyglucose uptake in the thoracic ninth vertebra with a suspicious enhancing lesion in the central spinal canal but without significant bony cortical disruption.

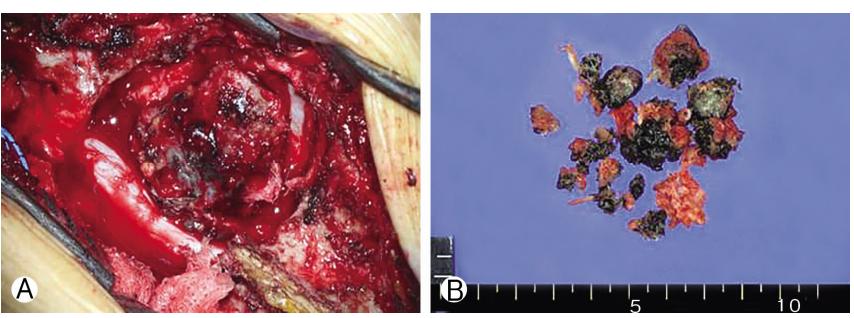

Fig. 4. Preoperative gross findings (A) and postoperative gross findings (B) of pigmented malignant melanoma on the thoracic ninth level, consisting of multiple pieces of pinkish tan tissue.

A 49-year-old man was diagnosed with primary spinal intramedullary malignant melanoma 18 years before recurrent disease was detected ${ }^{9}$. At diagnosis, the cancer was partially removed, and local radiation therapy up to $50 \mathrm{cGy}$ was performed. Most malignant melanomas are diagnosed at an early stage, when surgical excision can be curative; therefore, proper surgical resection is critical for initial management in order to maximize long-term survival ${ }^{3,12,13)}$. However, some patients have metastatic disease at presentation, and some develop metastases after initial definitive treatment. There are established treatments for advanced malignant melanoma including surgical metastasectomy, immunotherapy, targeted therapy (mitogen-activated protein kinase pathway [MARK] inhibition, KIT mutation), radiation therapy, and cytotoxic chemotherapy. The primary systemic therapy approaches for patients with metastatic melanoma are checkpoint-inhibitor immunotherapy and targeted therapy against the $\mathrm{MAPK}^{1,8)}$. Although cytotoxic chemotherapy was widely used prior to the development of checkpoint inhibition immunotherapy and targeted therapies, it does not have an established role in patients with metastatic melanoma, ${ }^{2,6}$. Radiotherapy can be a useful treatment option for patients with melanoma in some settings. It can provide effective palliation for the $40 \%$ to $50 \%$ of patients who develop unresectable, locally recurrent, or symptomatic metastatic disease including bone pain, epidural spinal cord compression, or central nervous system symptoms ${ }^{5,11)}$. The factors that allow long-term survival in rare malignant melanoma patients are not entirely clear. In those cases, the possibility of 


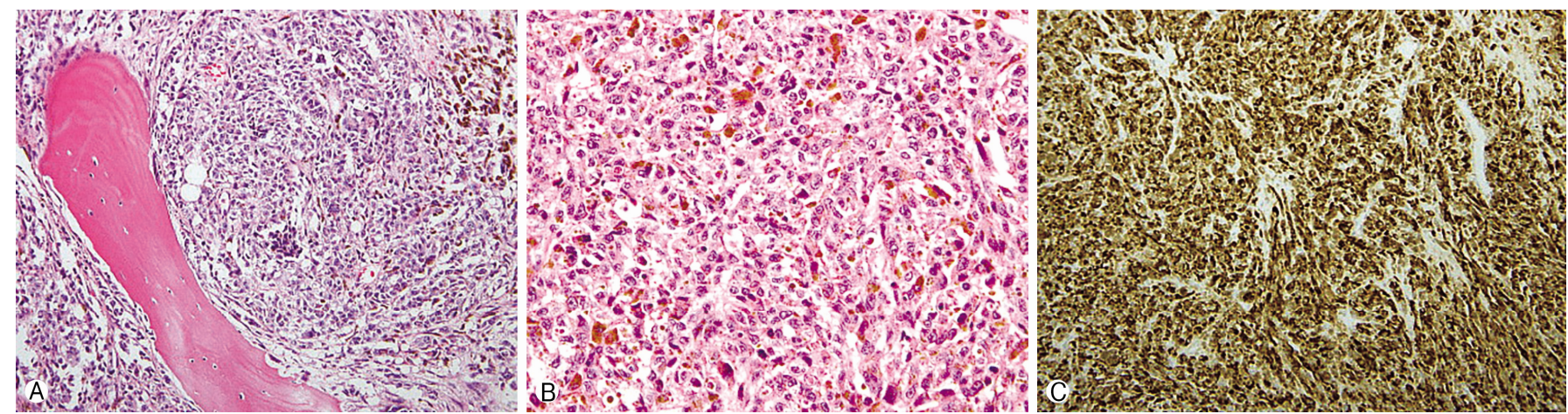

Fig. 5. (A, B) Histological and immunohistochemical findings. Pleomorphic cells with large vesicular nuclei, prominent nucleoli, and plentiful mitoses (H\&E: A, $\times 200 ; B, \times 400)$. (C) Diffuse strong cytoplasmic staining with HMB45 immunohistochemistry $(\times 200)$.
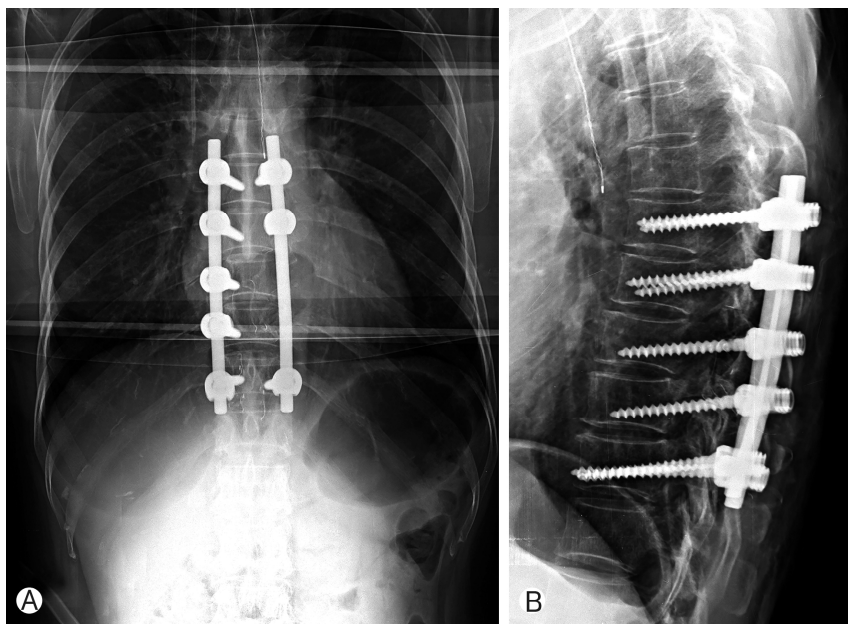

Fig. 6. Postoperative thoracic spine X-ray anterior-posterior (A), lateral (B). Total laminectomy T9, costotransversectomy T9 (left), pedicle resection T9 (left), T10 (left), total removal of tumor, pedicle screw fixation T7-8-9-10-11 (right), T7-8-11 (left), posterolateral fusion with allobone chips.

long-term survival without metastasis was observed after surgical resection of primary malignant melanoma, but multiple metastases were identified after detection of the first metastatic lesion. This suggests that metastasis in malignant melanoma patients cannot be explained simply with basic factors such as hematogenous metastasis or primary lesion size.

\section{CONCLUSION}

Malignant melanoma needs to be removed at first diagnosis in order to increase long-term survival because of the high rates of multiple metastases. After removal of malignant melanoma, lifelong screening should be performed despite the absence of recurrence or metastasis. Comprehensive factors have to be included in studies on long-term survival without metastasis or recurrence.

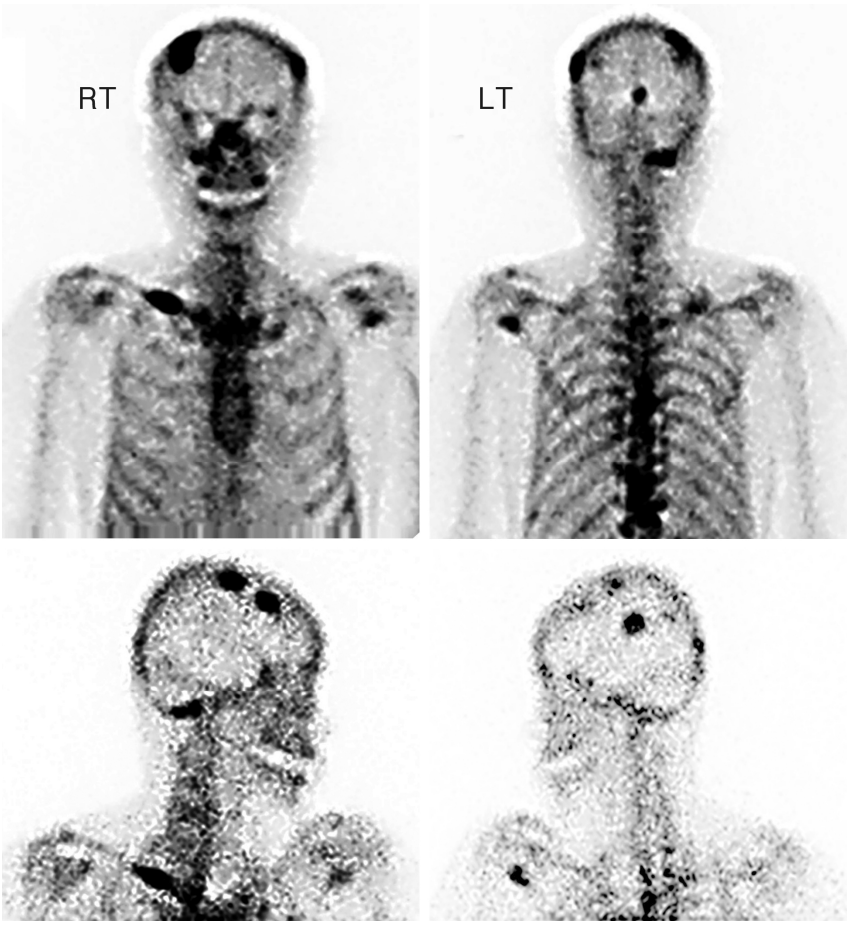

Fig. 7. Whole body bone scan seven months after resection of the vertebral body lesion. Newly developed multiple bony uptake at skull, right clavicle, left scapula, and thoracic vertebrae, signifying probable progression of bony metastases.

\section{CONFLICT OF INTEREST}

No potential conflict of interest relevant to this article was reported.

\section{REFERENCES}

1. Ackerman A, Klein O, McDermott DF, Wang W, Ibrahim N, Lawrence DP, et al: Outcomes of patients with metastatic melanoma treated with immunotherapy prior to or after BRAF inhibitors. 
Cancer 120:1695-1701, 2014

2. Atkins MB: The Role of Cytotoxic Chemotherapeutic Agents Either Alone or in Combination With Biological Response Modifiers. in Kirkwood JK (ed). Molecular Diagnosis, Prevention \& Therapy of Melanoma. New York: Marcel Dekker, pp219-251, 1997

3. Cascinelli $\mathrm{N}$ : Margin of resection in the management of primary melanoma. Semin Surg Oncol 14:272-275, 1998

4. Chew AL, Spilsbury K, Isaacs TW: Survival from uveal melanoma in Western Australia 1981-2005. Clin Exp Ophthalmol 43:422428, 2015

5. Guadagnolo BA, Prieto V, Weber R, Ross MI, Zagars GK: The role of adjuvant radiotherapy in the local management of desmoplastic melanoma. Cancer 120:1361-1368, 2014

6. Houghton AN, Legha S, Bajorin DF: Chemotherapy for Metastatic Melanoma. in Balch CM, Houghton AN, Milton GW, Sober AJ, Soong SJ (eds). Cutaneous Melanoma, ed 2. Philadelphia (PA): JB Lippincott Company, pp498, 1992

7. Kohler BA, Sherman RL, Howlader N, Jemal A, Ryerson AB, Henry KA, et al: Annual report to the Nation on the Status of Cancer, 1975-2011, featuring incidence of breast cancer subtypes by race/ ethnicity, poverty, and state. J Natl Cancer Inst 107:djv048, 2015

8. Larkin J, Chiarion-Sileni V, Gonzalez R, Grob JJ, Cowey CL, Lao $\mathrm{CD}$, et al: Combined nivolumab and ipilimumab or monotherapy in untreated melanoma. N Engl J Med 373:23-34, 2015

9. Nishihara M, Sasayama T, Kondoh T, Tanaka K, Kohmura E, Kudo $\mathrm{H}$ : Long-term survival after surgical resection of primary spinal malignant melanoma. Neurol Med Chir (Tokyo) 49:546-548, 2009

10. Seddon JM, Gragoudas ES, Egan KM, Glynn RJ, Howard S, Fante $\mathrm{RG}$, et al: Relative survival rates after alternative therapies for uveal melanoma. Ophthalmology 97:769-777, 1990

11. Strom T, Caudell JJ, Han D, Zager JS, Yu D, Cruse CW, et al: Radiotherapy influences local control in patients with desmoplastic melanoma. Cancer 120:1369-1378, 2014

12. Veronesi U, Cascinelli N: Narrow excision (1-cm margin). A safe procedure for thin cutaneous melanoma. Arch Surg 126:438-441, 1991

13. Veronesi U, Cascinelli N, Adamus J, Balch C, Bandiera D, Barchuk A, et al: Thin stage I primary cutaneous malignant melanoma. Comparison of excision with margins of 1 or $3 \mathrm{~cm}$. N Engl J Med 318: 1159-1162, 1988 